\title{
Avaliação biométrica de tangelo Page e pomelo Flame sobre diferentes porta-enxertos no semiárido do Ceará, Brasil
}

\author{
Kassio Ewerton Santos Sombra ${ }^{1 *}$, Francisco Leandro Costa Loureiro ${ }^{2}$, \\ Alexandre Caique Costa e Silva ${ }^{3}$, Marcio Porfirio da Silva ${ }^{1}$, Orlando Sampaio Passos ${ }^{4}$ \\ $\&$ Debora Costa Bastos ${ }^{4}$
}

\section{RESUMO}

O cultivo de citros ainda apresenta como uma de suas vulnerabilidades a baixa diversificação de variedades-copas e porta-enxertos, como constata-se nos principais polos citrícolas, onde predominam as laranjas Pêra, Valência e Natal (Citrus sinensis L. Osbeck), geralmente, enxertadas sobre clones nucelares de limão Cravo (C. limonia Osbeck), que têm sido largamente recomendados por sua alta compatibilidade, e capacidade de inferir precocidade e produtividade à copa, além de resistência à seca e ao vírus causador da tristeza. Entretanto, o limão Cravo apresenta susceptibilidade aos fungos causadores da gomose (Phytophthora spp.) e da verrugose (Elsinoe fawcettii), ao declínio e a morte súbita dos citros (MSC), além de intolerância aos viróides da exocorte e xiloporose. Sendo assim, diversificar pode elevar a segurança da cadeia produtiva, assim como, disponibilizar uma maior variedade de frutos cítricos ao consumidor, e potencialmente, elevar a rentabilidade por unidade produtiva. Diante disto, o trabalho teve objetivo de avaliar o desenvolvimento inicial de tangelo Page [C. clementina hort. ex Tanaka $\times(C$. paradisi Macfad. $\times C$. tangerina Tanaka) e pomelo Flame (C. paradisi Mcf.) sobre diferentes porta-enxertos cítricos através de avaliações biométricas periódicas. As combinações entre tangelo Page e os citrandarins Indio e Riverside, e do pomelo Flame sobre tangerina Sunki tropical, e os citrandarins San Diego e Indio, tiveram os desenvolvimentos iniciais mais elevados, demonstrando vigor considerável diante das condições edafoclimáticas do semiárido cearense, diferindo estatisticamente das demais combinações para altura, diâmetro e volume de copa, com alta afinidade entre copa e porta-enxerto, sendo alternativas para diversificar o cultivo de citros em condições similares.

Termos de indexação: citricultura, desenvolvimento, diversificação, genótipos.

\section{Biometric evaluation of Page tangelo and Flame grapefruit on different rootstocks in the Semi-arid of Ceará, Brazil}

\section{SUMMARY}

Citrus cultivation still presents as one of its vulnerabilities the low diversification of crown varieties and rootstocks, as it is observed in the main citrus poles, where the Pêra, Valencia and Natal oranges (Citrus sinensis L. Osbeck) predominate, generally, grafted on Rangpur lemon

\footnotetext{
${ }^{1}$ Departamento de Fitotecnia, Universidade Federal do Ceará - UFC, Fortaleza, CE, Brasil

2 Departamento de Fitotecnia, Universidade Federal Rural do Semiárido - UFERSA, Mossoró, RN, Brasil

${ }^{3}$ Departamento de Agronomia, Instituto Federal de Educação, Ciência e Tecnologia do Ceará - IFCE, Fortaleza, CE, Brasil

${ }^{4}$ Empresa Brasileira de Pesquisa Agropecuária - EMBRAPA, Cruz das Almas, BA, Brasil

*Autor correspondente: Kássio Ewerton Santos Sombra, Departamento de Fitotecnia, Universidade Federal do Ceará - UFC, Av. Mister Hull, 2977, Bloco 805, Campus do Pici, CEP 60356001, Fortaleza, CE, Brasil. E-mail: kassiosombra@gmail.com
} 
(C. limonia Osbeck), which have been widely recommended for their high compatibility, and ability to infer precocity and productivity to the crown, as well as resistance to drought and the citrus tristeza virus (CTV). However, Rangpur lemon presents susceptibility to fungi causing gummosis (Phytophthora spp.) and citrus scab (Elsinoe fawcettii), to citrus decline and sudden death (MSC), besides intolerance to exocortis viroids and xyloporosis. Therefore, diversification can increase the security of the productive chain, as well as, provide a greater variety of citrus fruits to the consumer, and potentially increase profitability per unit of production. In view of this, the work had the objective of evaluating the initial development of Page tangelo [C. clementine hort. ex Tanaka $\mathrm{x}$ (C. paradisi Macfad $\times$ C. tangerina Tanaka)] and Flame pomelo ( $C$. paradisi $\mathrm{Mcf}$.) on different citrus rootstocks through periodic biometric evaluations. The combinations between tangelo Page and the Indio and Riverside citrandarins, and the Flame pomelo on tropical Sunki tangerine, and San Diego and Indio citrandarins, had the highest initial development, demonstrating considerable force in the soil and climatic conditions of the semi-arid region of Ceará, differing statistically from other combinations for height, diameter and crown volume, with high affinity between canopy and rootstock, being alternatives to diversify citrus cultivation under similar conditions.

Index terms: citriculture, development, diversification, genotypes.

\section{INTRODUÇÃO}

Os citros consolidaram-se entre as espécies mais cultivadas no mundo, principalmente, diante da importância da laranja doce (C. sinensis (L.) Osbeck), consumida geralmente 'in natura', ou como suco de laranja concentrado congelado (SLCC) (Cunha Sobrinho et al., 2013). Porém, além das laranjas, os citros compreendem outras plantas do gênero Citrus, e de gêneros afins, como, por exemplo, Fortunella e Poncirus, com destaque para as tangerinas (Citrus reticulata Blanco ou $C$. deliciosa Tenore), os limões (C. limon L. Burm. f. pro. sp. ou C. aurantifolia Christm. Swingle pro. sp.), limas ácidas, como o Tahiti (C. latifolia Tanaka), e doces, como a lima da Pérsia (C. limettioides Tanaka), além de, pomelos (C. maxima Burm. f. Merr.), cidras (C. medica L.) e toranjas (C. paradisi Macfad. pro sp.) (Barbieri \& Stumpf, 2008; Silva et al., 2016; Passos et al., 2016).

Apesar da diversidade existente, o cultivo de citros ainda apresenta a baixa diversificação de variedades copa e porta-enxertos como uma de suas vulnerabilidades, como constata-se nos principais polos citrícolas (Lopes et al., 2011), onde predominam, por exemplo, copas de laranjas Pêra, Valência e Natal, geralmente, enxertadas sobre clones de limão Cravo (C. limonia Osbeck) (CDA, 2017). Entretanto, diversificar o uso de copas, e de porta-enxertos, pode elevar a segurança da cadeia produtiva, assim como, disponibilizar uma maior variedade de frutos cítricos ao consumidor, e potencialmente, elevar a rentabilidade por unidade produtiva (Cunha Sobrinho et al., 2013).

Entre as copas promissoras, alguns híbridos, como o tangelo Page [Citrus clementina hort. ex Tanaka $\times(C$. paradisi $\mathrm{Mcf} \times C$. tangerina Tanaka) $]($ Passos $\&$ Soares Filho, 2003), e o pomelo Flame (C. paradisi)
(Bastos et al., 2017a) podem ser alternativas para a citricultura no semiárido, atendendo o mercado frutos de mesa e/ou indústria (Passos et al., 2005; Bastos et al., 2014; Carvalho et al., 2016).

O tangelo Page é um híbrido interespecífico, obtido a partir do cruzamento entre tangerina Clementina ( $C$. clementina) e tangelo Minneola ( $C$. paradisi $\times C$. tangerina), que geralmente, apresenta porte médio e copa arredondada, e produz frutos pequenos e sucosos, com até cinco sementes em média, casca e polpa de cores alaranjadas (Passos et al., 2005; Bastos et al., 2014). Já o pomelo Flame (C. paradisi) possui, geralmente, copas maiores, de formato arredondado e crescimento aberto, resultantes do vigor elevado, produzindo frutos de tamanho grande e formato arredondado, com casca fina e lisa de coloração laranja e manchas vermelhas, que reveste a polpa firme de coloração vermelho intensa, com poucas ou nenhuma semente, elevados teores de vitamina $\mathrm{C}$, fibras e antioxidantes (Oliveira et al., 2012; Bastos et al., 2017a).

Entre os porta-enxertos promissores, os citrandarins Índio, Riverside e San Diego (Citrus sunki Hayata hort. ex Tanaka $\times$ Poncirus trifoliata $\mathrm{L}$. Raf.) vêm destacando-se (Bastos et al., 2017b; Bastos et al., 2017c), principalmente, por apresentar adaptabilidade às condições tropicais, compatibilidade com a maioria das variedades-copas, equiparando-se, por exemplo, ao limão Cravo, além de possuir resistência aos fungos causadores da gomose (Phytophthora spp.) e a morte súbita dos citros (Passos et al., 2011a, 2011b, 2011c; Passos, 2012).

Diante disto, o trabalho teve objetivo de avaliar o desenvolvimento vegetativo inicial de tangelo Page e pomelo Flame sobre diferentes porta-enxertos cítricos, tolerantes ou resistentes aos principais estresses bióticos e abióticos em condições semiáridas, através de avaliações biométricas periódicas. 


\section{MATERIAL E MÉTODOS}

O experimento foi desenvolvido entre 2013 e 2016, na Área Experimental Nilson Mendonça, localizada no Sítio Pau Branco, zona rural do município de Russas, Ceará, Brasil (4 53’ 0.10” S; $37^{\circ} 55^{\prime} 1.20^{\prime}$ ” O, altitude de $19 \mathrm{~m}$ ) (Figura 1).

Russas é um munícipio localizado na microrregião do Baixo Jaguaribe, no semiárido do Ceará, de clima seco e muito quente, tipo BSw'h' (Köppen) (Alvares et al., 2013). A temperatura média anual é de cerca de $27,1^{\circ} \mathrm{C}$, oscilando entre aproximadamente $22,3{ }^{\circ} \mathrm{C}$ de temperatura mínima, e $34,8^{\circ} \mathrm{C}$ de máxima, com umidade relativa do ar (UR) oscilando entre $44 \%$ e $85 \%$, e regime pluviométrico médio de 857,7 mm, variando entre 548 e 992 mm, 'mal' distribuídos ao longo do ano.

O delineamento utilizado foi inteiramente casualizado (DIC), esquema $2 \times 6(n=48)$, avaliando o desenvolvimento vegetativo inicial de combinações copa/porta-enxerto entre tangelo Page, ou pomelo Flame e os porta-enxertos: limão Cravo Santa Cruz; citrandarins San Diego, Indio e Riverside; híbrido 059 [C. sunki $\times($ C. limonia $\times$ P. trifoliata $)]$ e tangerina Sunki Tropical (C. sunki hort). Implantando-se quatro repetições (mudas) por combinação.

As mudas cítricas foram produzidas por enxertia (borbulhia) e disponibilizadas na forma de muda no torrão pela Embrapa Semiárido, através do Banco Ativo de Germoplasma de Citros da Embrapa Mandioca e Fruticultura, situada em Cruz das Almas - Bahia, Brasil.

O solo da área experimental, classificado como Vertissolo Hidromórfico Órtico Típico (SiBCS) (Embrapa, 2013), foi mecanizado e corrigido, e posteriormente, realizou-se a marcação das covas, cavadas com aproximadamente $40 \mathrm{~cm}$ de diâmetro e $40 \mathrm{~cm}$ de profundidade, sob o espaçamento adensado de 5 metros entre linhas e 2 metros entre plantas. Em seguida, realizou-se a adubação de fundação, utilizando composto orgânico, produzido a partir de bagana (palha triturada) de carnaúba (Copernicia prunifera Mill. H. E. Moore) (Arecaceae) e esterco bovino curtido, numa proporção de 20 litros por cova (Primavesi, 2014; Sombra et al., 2018).

Após sete dias, as mudas foram transplantadas, suprindo-se a demanda hídrica da cultura através de irrigação localizada adaptada utilizando microaspersores, concentrando-se as maiores lâminas nos meses mais quentes do ano.

O desenvolvimento vegetativo das combinações foi obtido a partir de avaliações biométricas realizadas aos $18,24,30$ e 36 meses após o transplantio, nas quais mediam-se: a altura (h), do colo ao ápice; e os diâmetros da copa, nos sentidos entre plantas e entre linhas (Dle Dr), utilizando trena milimetrada; e os diâmetros do enxerto e do porta-enxerto, tomados $10 \mathrm{~cm}$ abaixo e acima do ponto de enxertia das combinações, utilizando paquímetro digital

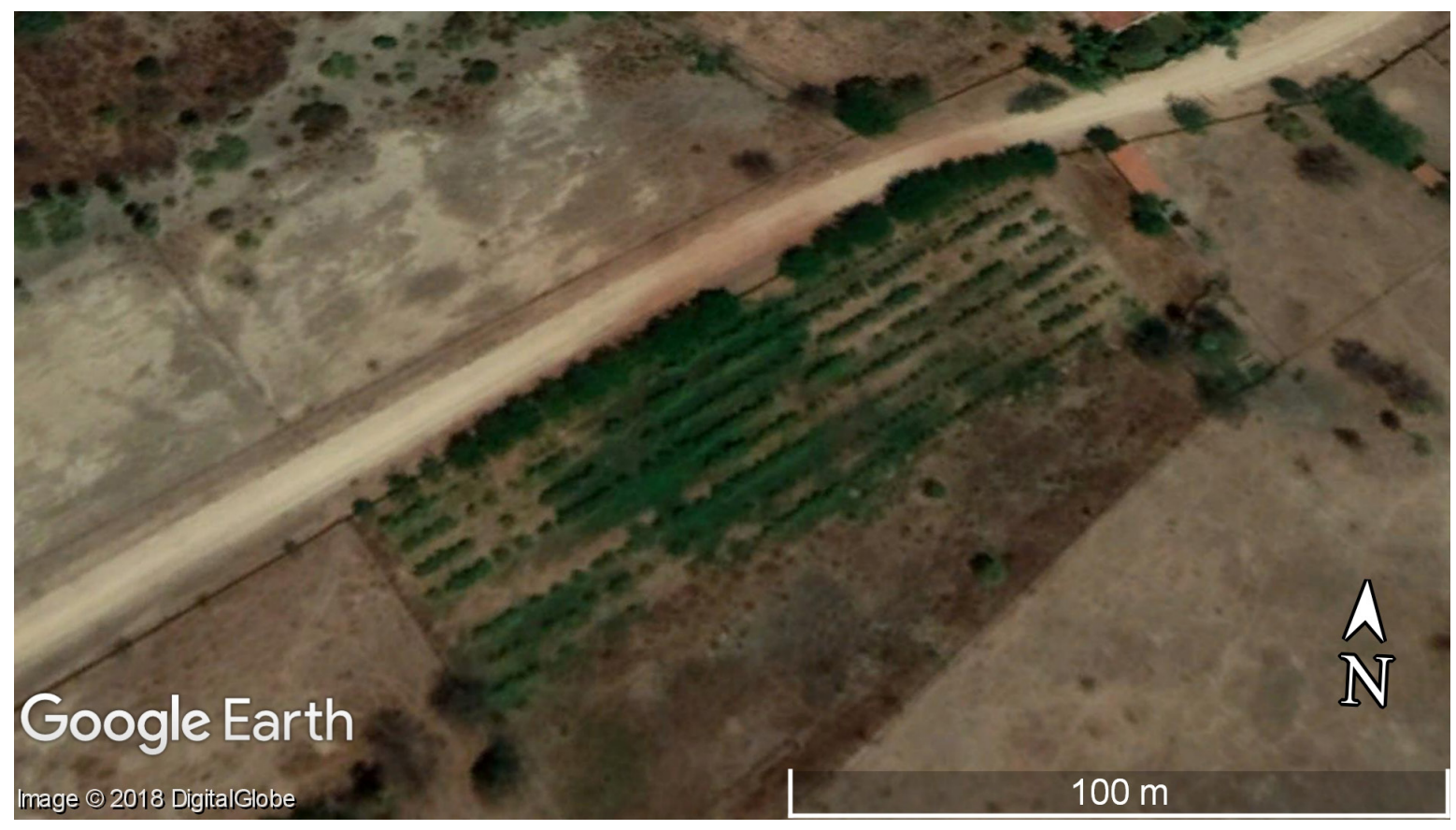

Figura 1. Área Experimental Nilson Mendonça, Sítio Pau Branco, Russas - CE, Brasil. Fonte: Google Earth Pro 7.1.5.1557. Data da imagem: 29/12/2016. 
$(0,01 \mathrm{~mm})$. Os valores foram utilizados para cálculo do volume de copa $\left(\mathrm{V}^{3}\right)$, aplicando-se a fórmula descrita por Fallahi \& Rodney (1991):

$V^{3}=(\pi / 6) \times H \times D l \times D r$

Onde: $\mathrm{V}=$ volume médio da copa; $\mathrm{H}=$ altura da planta; $\mathrm{Dl}$ = diâmetro da copa no sentido entre linhas; $\mathrm{e} \mathrm{Dr}$ = diâmetro da copa no sentido entre plantas.

Já a razão de compatibilidade (IC) das combinações foi calculada utilizando a formula descrita a seguir:

$I C=d / D$

Onde $=\mathrm{IC}=$ índice de compatibilidade copa/porta-enxerto; $\mathrm{d}=$ diâmetro do enxerto; $\mathrm{e} \mathrm{D}$ = diâmetro do porta-enxerto. Sendo a compatibilidade plena equivalente a 1 (Simonetti, 2015; Rodrigues et al., 2016).

Os dados obtidos foram submetidos à análise de variância (ANOVA), e nos casos de diferença significativa, utilizou-se o Teste de Scott-Knott ao nível de 5\% de probabilidade $(\mathrm{p} \leq 0,05)$ para comparação de médias, utilizando-se software estatístico ASSISTAT ${ }^{\circledR}$ (Silva \& Azevedo, 2016).

\section{RESULTADOS E DISCUSSÃO}

Os porta-enxertos apresentaram compatibilidade satisfatória com as duas copas, registrando-se valores de razão de compatibilidade (IC) superiores a ' 0,81 ' em todas as combinações com pomelo Flame, e de no mínimo ‘ 0,70 ’ nas combinações com tangelo Page (Tabela 1).

Rodrigues et al. (2016) obtiveram valores similares de razão de compatibilidade entre combinações de quatro copas e catorze porta-enxertos, registrando IC médio de '0,72', assim como, Bastos et al. (2017b), que obtiveram, por exemplo, valores de 0,89 de compatibilidade entre laranja Valência Tuxpan e limão Cravo Santa Cruz, 0,84 entre laranja Pêra-D6 e citrandarin Indio, e 0,77 entre laranja Rubi e citrandarin Riverside. Sombra et al. (2017a) obtiveram 0,73 de IC entre combinações de tangor Piemonte e tanga Sunki Tropical (porta-enxerto), e 0,93 entre limão Siciliano e limão Cravo Santa Cruz, o que indica alta afinidade entre as copas e os porta-enxertos, sendo 1 a compatibilidade plena (Cunha Sobrinho et al., 2013; Sombra et al. 2017b).

A altura (h) das plantas de pomelo Flame só começou a diferir estatisticamente entre os porta-enxertos a partir dos 24 meses, destacando-se a altura das combinações com citrandarins Indio, Riverside e San Diego, além do limão Cravo Santa Cruz, que predominaram até os 36 meses após o transplantio, com altura final de 2,13; 1,90; 2,10 e 2,37 metros, respectivamente (Tabela 2).

Ao comparar os resultados obtidos pelo pomelo Flame, com os do tangelo Page, constata-se que apesar das características das duas copas serem distintas, principalmente o vigor relatado na literatura (Passos \& Soares Filho, 2003; Bastos et al., 2017a), o tangelo Page obteve valores estatisticamente similares sobre os citrandarins Indio e Riverside, assim como o limão Cravo Santa Cruz, com altura de 2,08; 1,94 e 2,07, respectivamente, aos 36 meses. Porém, o tangelo atingiu altura inferior quando enxertada sobre citrandarin San Diego.

Bastos et al. (2017b) obtiveram alturas similares para combinações entre as laranjas Valência Tuxpan, Pêra D-6 e Rubi, sobre os citrandarins, assim como, Bastos et al. (2017c) obtiveram valores superiores ao do tangelo Page, e similares ao do pomelo Flame, avaliando combinações de lima ácida Tahiti e os citrandarins Indio e Riverside, além do limão Cravo Santa Cruz, com altura de 2,12; 2,00 e 2,12 m, respectivamente, aos 30 meses após o transplantio. Já Sombra et al. (2017a) obtiveram plantas de tangor Piemonte com 2,10 e 1,91 m, também sobre os citrandarins Indio e Riverside.

As combinações que apresentaram maiores alturas, e consequentemente, tinham maior vigor, obtiveram copas com maior diâmetro (Tabela 3) e volume $\left(\mathrm{V}^{3}\right)$ (Tabela 4).

Tabela 1. Razão de Compatibilidade (IC) das combinações aos 36 meses após o transplantio, Russas-CE, Brasil, 2018

\begin{tabular}{lc}
\hline \multicolumn{1}{c}{ Combinação copa $\times$ porta-enxerto } & IC \\
\hline Tangelo Page $\times$ limão Cravo Santa Cruz & $0,77 \mathrm{a}$ \\
Tangelo Page $\times$ citrandarin San Diego & $0,70 \mathrm{~b}$ \\
Tangelo Page $\times$ citrandarin Indio & $0,78 \mathrm{a}$ \\
Tangelo Page $\times$ citrandarin Riverside & $0,79 \mathrm{a}$ \\
Tangelo Page $\times$ híbrido 059 & $0,75 \mathrm{a}$ \\
Tangelo Page $\times$ tangerina Sunki Tropical & $0,81 \mathrm{a}$ \\
Pomelo Flame $\times$ limão Cravo Santa Cruz & $0,94 \mathrm{a}$ \\
Pomelo Flame $\times$ citrandarin San Diego & $0,82 \mathrm{a}$ \\
Pomelo Flame $\times$ citrandarin Indio & $0,84 \mathrm{a}$ \\
Pomelo Flame $\times$ citrandarin Riverside & $0,82 \mathrm{a}$ \\
Pomelo Flame $\times$ citrumelo Swingle 4475 & $0,88 \mathrm{a}$ \\
Pomelo Flame $\times$ tangerina Sunki Tropical & $0,86 \mathrm{a}$ \\
C. V. $(\%)$ & 10,03 \\
Valor F & $2,4212 *$ \\
Valor $\mathrm{p}$ & 0,0225 \\
\hline As
\end{tabular}

As médias seguidas pela mesma letra não diferem estatisticamente entre si. *significativo ao nível de $5 \%$ de probabilidade $(\mathrm{p}<0,05)$. 
Tabela 2. Altura (h) de tangelo Page e pomelo Flame sobre seis porta-enxertos aos 18, 24, 30 e 36 meses após o transplantio, Russas - CE, Brasil, 2018

\begin{tabular}{lcccc}
\hline \multicolumn{1}{c}{ Porta-enxerto } & 18 meses & 24 meses & 30 meses & 36 meses \\
\hline T. Page $\times$ limão Cravo Santa Cruz & $1,100 \mathrm{~b}$ & $1,505 \mathrm{~b}$ & $1,782 \mathrm{~b}$ & $2,075 \mathrm{a}$ \\
T. Page $\times$ citrandarin San Diego & $0,937 \mathrm{~b}$ & $1,220 \mathrm{~d}$ & $1,387 \mathrm{c}$ & $1,750 \mathrm{~b}$ \\
T. Page $\times$ citrandarin Indio & $1,070 \mathrm{~b}$ & $1,480 \mathrm{~b}$ & $1,755 \mathrm{~b}$ & $2,085 \mathrm{a}$ \\
T. Page $\times$ citrandarin Riverside & $1,312 \mathrm{a}$ & $1,662 \mathrm{a}$ & $1,732 \mathrm{~b}$ & $1,947 \mathrm{a}$ \\
T. Page $\times$ híbrido 059 & $1,040 \mathrm{~b}$ & $1,112 \mathrm{~d}$ & $1,210 \mathrm{c}$ & $1,332 \mathrm{~b}$ \\
T. Page $\times$ tangerina Sunki Tropical & $0,972 \mathrm{~b}$ & $1,147 \mathrm{~b}$ & $1,275 \mathrm{c}$ & $1,435 \mathrm{~b}$ \\
P. Flame $\times$ limão Cravo Santa Cruz & $1,335 \mathrm{a}$ & $1,772 \mathrm{a}$ & $2,002 \mathrm{a}$ & $2,370 \mathrm{a}$ \\
P. Flame $\times$ citrandarin San Diego & $1,262 \mathrm{a}$ & $1,720 \mathrm{a}$ & $1,695 \mathrm{~b}$ & $2,102 \mathrm{a}$ \\
P. Flame $\times$ citrandarin Indio & $1,210 \mathrm{a}$ & $1,767 \mathrm{a}$ & $1,907 \mathrm{a}$ & $2,135 \mathrm{a}$ \\
P. Flame $\times$ citrandarin Riverside & $1,215 \mathrm{a}$ & $1,715 \mathrm{a}$ & $1,687 \mathrm{~b}$ & $1,905 \mathrm{a}$ \\
P. Flame $\times$ citrumelo Swingle 4475 & $1,237 \mathrm{a}$ & $1,427 \mathrm{c}$ & $1,485 \mathrm{c}$ & $1,587 \mathrm{~b}$ \\
P. Flame $\times$ tangerina Sunki Tropical & $1,410 \mathrm{a}$ & $1,775 \mathrm{a}$ & $1,967 \mathrm{a}$ & $2,255 \mathrm{a}$ \\
C.V. $(\%)$ & 14,74 & 10,06 & 10,71 & 12,24 \\
Valor de F & $2,983 *$ & $10,702 *$ & $8,814 *$ & $7,714 *$ \\
\hline
\end{tabular}

As médias seguidas pela mesma letra não diferem estatisticamente entre si. *significativo ao nível de $1 \%$ de probabilidade $(\mathrm{p}<0,01)$.

Tabela 3. Diâmetro de copa (D) de tangelo Page e pomelo Flame sobre seis porta-enxertos aos 18, 24, 30 e 36 meses após o transplantio, Russas - CE, Brasil, 2018

\begin{tabular}{lcccc}
\hline \multicolumn{1}{c}{ Porta-enxerto } & $18 \mathrm{meses}$ & $24 \mathrm{meses}$ & $30 \mathrm{meses}$ & 36 meses \\
\hline T. Page $\times$ limão Cravo Santa Cruz & $0,878 \mathrm{~b}$ & $1,307 \mathrm{~b}$ & $1,543 \mathrm{~b}$ & $2,002 \mathrm{~b}$ \\
T. Page $\times$ citrandarin San Diego & $0,686 \mathrm{~b}$ & $0,951 \mathrm{c}$ & $1,343 \mathrm{c}$ & $1,670 \mathrm{c}$ \\
T. Page $\times$ citrandarin Indio & $0,818 \mathrm{~b}$ & $1,326 \mathrm{~b}$ & $1,422 \mathrm{c}$ & $2,055 \mathrm{~b}$ \\
T. Page $\times$ citrandarin Riverside & $1,083 \mathrm{a}$ & $1,646 \mathrm{a}$ & $1,606 \mathrm{~b}$ & $1,906 \mathrm{~b}$ \\
T. Page $\times$ híbrido 059 & $0,608 \mathrm{~b}$ & $0,858 \mathrm{c}$ & $1,016 \mathrm{~d}$ & $1,320 \mathrm{~d}$ \\
T. Page $\times$ tangerina Sunki Tropical & $0,572 \mathrm{~b}$ & $0,911 \mathrm{c}$ & $0,995 \mathrm{~d}$ & $1,297 \mathrm{~d}$ \\
P. Flame $\times$ limão Cravo Santa Cruz & $1,172 \mathrm{a}$ & $1,730 \mathrm{a}$ & $1,927 \mathrm{a}$ & $2,486 \mathrm{a}$ \\
P. Flame $\times$ citrandarin San Diego & $1,255 \mathrm{a}$ & $1,578 \mathrm{a}$ & $1,675 \mathrm{~b}$ & $2,190 \mathrm{~b}$ \\
P. Flame $\times$ citrandarin Indio & $1,260 \mathrm{a}$ & $1,717 \mathrm{a}$ & $1,916 \mathrm{a}$ & $2,372 \mathrm{a}$ \\
P. Flame $\times$ citrandarin Riverside & $1,120 \mathrm{a}$ & $1,661 \mathrm{a}$ & $1,680 \mathrm{~b}$ & $2,035 \mathrm{~b}$ \\
P. Flame $\times$ citrumelo Swingle 4475 & $1,075 \mathrm{a}$ & $1,352 \mathrm{~b}$ & $1,463 \mathrm{c}$ & $1,698 \mathrm{c}$ \\
P. Flame $\times$ tangerina Sunki Tropical & $1,392 \mathrm{a}$ & $1,735 \mathrm{a}$ & $1,853 \mathrm{a}$ & $2,337 \mathrm{a}$ \\
C.V. $(\%)$ & 19,34 & 13,86 & 12,72 & 12,91 \\
Valor de F & $8,120 *$ & $11,923 *$ & $10.090 *$ & $9.543 *$ \\
\hline
\end{tabular}

As médias seguidas pela mesma letra não diferem estatisticamente entre si. *significativo ao nível de $1 \%$ de probabilidade $(\mathrm{p}<0,01)$.

As plantas de pomelo Flame sobre o citrandarin Indio, limão Cravo Santa Cruz e tangerina Sunki Tropical, apresentaram as copas com maiores diâmetros e volumes, citando-se, copas com 2,37 m de diâmetro e $6,58 \mathrm{~m}^{3}$ de volume da combinação entre pomelo Flame e citrandarin Indio, aos 36 meses após o transplantio. Já as plantas de tangelo Page apresentam valores inferiores aos dos pomelos, devido as características e ao vigor distinto das variedades copa, porém, destacaram-se as combinações entre tangelo Page e os citrandarins Indio e Riverside, além do, limão Cravo Santa Cruz, com valores de 2,05;1,90 e 2,02 m de diâmetro de copa, e 4,60; 3,82 e 4,60 $\mathrm{m}^{3}$ de volume de copa (Figuras 2 e 3), respectivamente (Tabela 4).

Sombra et al. (2017a) obtiveram valores inferiores de diâmetro e volume de copa utilizando a tangor Piemonte como copa, o que, possivelmente, deve-se as características 
Tabela 4. Volume de copa $\left(\mathrm{m}^{3}\right)$ de tangelo Page e pomelo Flame sobre seis porta-enxertos aos 18, 24, 30 e 36 meses após o transplantio, Russas - CE, Brasil, 2018

\begin{tabular}{lcccc}
\hline \multicolumn{1}{c}{ Porta-enxerto } & 18 meses & 24 meses & 30 meses & 36 meses \\
\hline T. Page $\times$ limão Cravo Santa Cruz & $0,487 \mathrm{~b}$ & $1,477 \mathrm{~b}$ & $2,367 \mathrm{ba}$ & $4,604 \mathrm{~b}$ \\
T. Page $\times$ citrandarin San Diego & $0,234 \mathrm{~b}$ & $0,600 \mathrm{c}$ & $1,328 \mathrm{c}$ & $2,578 \mathrm{c}$ \\
T. Page $\times$ citrandarin Indio & $0,483 \mathrm{~b}$ & $1,368 \mathrm{~b}$ & $1,884 \mathrm{c}$ & $4,609 \mathrm{~b}$ \\
T. Page $\times$ citrandarin Riverside & $0,833 \mathrm{a}$ & $2,379 \mathrm{a}$ & $2,405 \mathrm{~b}$ & $3,827 \mathrm{c}$ \\
T. Page $\times$ híbrido 059 & $0,211 \mathrm{~b}$ & $0,462 \mathrm{c}$ & $0,659 \mathrm{c}$ & $1,236 \mathrm{c}$ \\
T. Page $\times$ tangerina Sunki Tropical & $0,194 \mathrm{~b}$ & $0,508 \mathrm{c}$ & $0,662 \mathrm{c}$ & $1,267 \mathrm{c}$ \\
P. Flame $\times$ limão Cravo Santa Cruz & $1,025 \mathrm{a}$ & $2,818 \mathrm{a}$ & $3,929 \mathrm{a}$ & $7,701 \mathrm{a}$ \\
P. Flame $\times$ citrandarin San Diego & $1,064 \mathrm{a}$ & $2,304 \mathrm{a}$ & $2,713 \mathrm{~b}$ & $5,672 \mathrm{~b}$ \\
P. Flame $\times$ citrandarin Indio & $1,005 \mathrm{a}$ & $2,754 \mathrm{a}$ & $3,734 \mathrm{a}$ & $6,582 \mathrm{a}$ \\
P. Flame $\times$ citrandarin Riverside & $0,842 \mathrm{a}$ & $2,551 \mathrm{a}$ & $2,630 \mathrm{~b}$ & $4,485 \mathrm{~b}$ \\
P. Flame $\times$ citrumelo Swingle 4475 & $0,761 \mathrm{a}$ & $1,379 \mathrm{~b}$ & $1,662 \mathrm{c}$ & $2,403 \mathrm{c}$ \\
P. Flame $\times$ tangerina Sunki Tropical & $1,466 \mathrm{a}$ & $2,787 \mathrm{a}$ & $3,544 \mathrm{a}$ & $6,483 \mathrm{a}$ \\
C.V. $(\%)$ & 42,56 & 33,35 & 33,69 & 37,10 \\
Valor de F & $6,346^{*}$ & $9,714 *$ & $8,205 *$ & $7,040^{*}$ \\
\hline
\end{tabular}

As médias seguidas pela mesma letra não diferem estatisticamente entre si. *significativo ao nível de $1 \%$ de probabilidade $(\mathrm{p}<0,01)$.

da variedade, porém, os maiores valores foram obtidos utilizando o limão Cravo Santa Cruz ou o citrandarin Indio como porta-enxertos, citando-se os valores finais de 2,02 e 1,77 m de diâmetro, além de 4,72 e 3,55 $\mathrm{m}^{3}$ de volume médio de copa, respectivamente, aos 36 meses após o transplantio.

Já o limão Siciliano, em estudo de Sombra et al. (2017b), apresentou maiores valores de diâmetro e volume de copa quando enxertado sobre os citrandarins Indio e Riverside, atingindo 2,26 e 2,28 $\mathrm{m}$ de diâmetro, e volume de copa de 6,55 e $6,59 \mathrm{~m}^{3}$, respectivamente, aos 36 meses. Os dois porta-enxertos também induziram os maiores valores a copa de Valência Tuxpan, em estudo de Bastos et al. (2017b), atingindo 1,66 e 1,62 $\mathrm{m}$ de diâmetro, e volume de 2,76 e $2,37 \mathrm{~m}^{3}$, respectivamente.

Analisando as curvas de desenvolvimento inicial das combinações copa/porta-enxertos, através das biometrias do volume de copa, (Figuras 1 e 2), constata-se que o limão Cravo Santa Cruz e o citrandarin Indio, induziram os desenvolvimentos iniciais mais significativos as copas de tangelo Page e pomelo Flame, que apresentaram maior vigor e precocidade, atingindo maiores valores de altura, diâmetro e volume de copas. Porém, diante dos riscos inerentes ao uso intenso de clones de limão Cravo (Lopes et al., 2011), a adoção do citrandarin Indio pode permitir que o produtor obtenha resultados similares em condições próximas ao do estudo, com a resistência ou tolerância à estresses bióticos e abióticos de importância econômica, inerentes a este porta-enxerto (Passos et al., 2011a).

A tangerineira Sunki Tropical e o Híbrido 059 induziram os menores valores de desenvolvimento ao tangelo Page, com volume de copa de 1,26 e 1,23 $\mathrm{m}^{3}$ aos 36 meses após o transplantio, respectivamente, apesar de não diferirem estatisticamente dos valores obtidos pelos citrandarins Riverside $\left(3,82 \mathrm{~m}^{3}\right)$ e San Diego $\left(2,57 \mathrm{~m}^{3}\right)$. Enquanto o citrumelo Swingle induziu os menores valores ao pomelo Flame, como por exemplo, altura de 1,58 m e volume de copa de $2,40 \mathrm{~m}^{3}$.

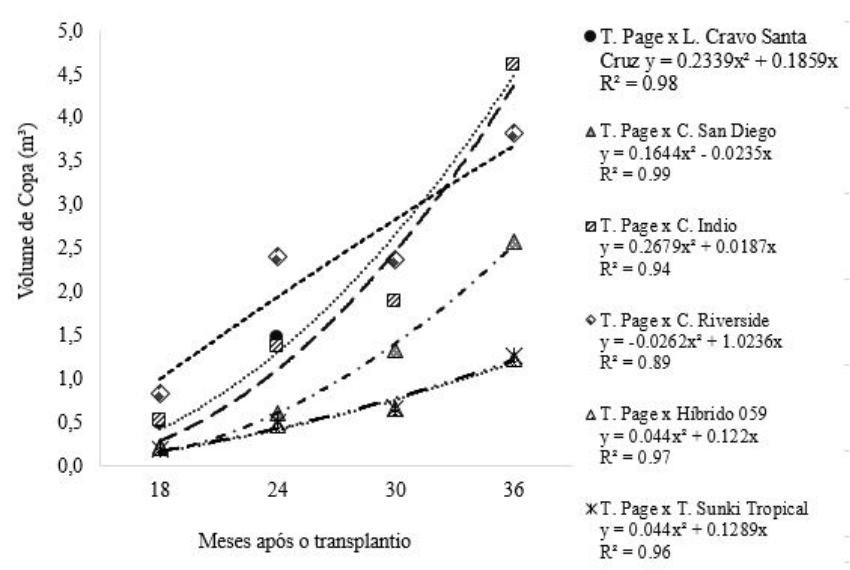

Figura 2. Volume de copa de tangelo Page sobre seis porta-enxertos ao longo dos 36 meses de desenvolvimento inicial após o transplantio, Russas - CE, Brasil. 2018. 


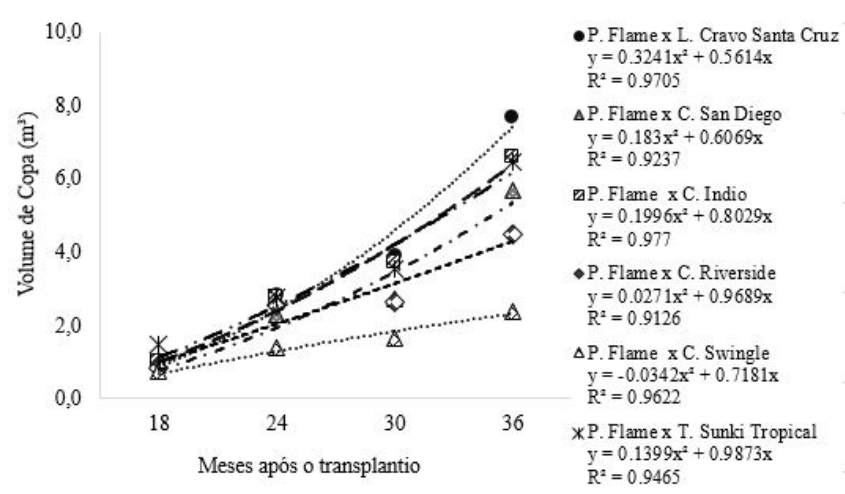

Figura 3. Volume de copa de pomelo Flame sobre seis porta-enxertos ao longo dos 36 meses de desenvolvimento inicial após o transplantio, Russas - CE, Brasil. 2018.

O menor desenvolvimento induzido por alguns portaenxertos, potencialmente semi-ananicantes ou ananicantes (Loureiro et al., 2016; Portella et al., 2016), pode tornar-se uma característica vantajosa, quando considerada a tendência de adensamento na citricultura moderna (Teófilo Sobrinho et al., 2012), que buscando o melhor aproveitamento dos recursos disponíveis (solo e água) e o aumento da produtividade, evoluiu de 250 plantas por hectare em 1980 (Azevedo et al., 2015), para 1000 plantas por hectare em 2018, necessitando de plantas com porte reduzido, que facilitem, por exemplo, a padronização dos equipamentos e dos tratos culturais, reduzindo os custos e melhorando a eficiência produtiva (Azevedo et al., 2015).

\section{CONCLUSÕES}

As combinações entre tangelo Page e os citrandarins Indio e Riverside, e do pomelo Flame sobre tangerina Sunki Tropical, e os citrandarins San Diego e Indio, tiveram os desenvolvimentos iniciais mais elevados, demonstrando vigor considerável diante das condições edafoclimáticas do semiárido cearense, e diferindo estatisticamente das demais combinação para altura, diâmetro e volume de copa, com alta afinidade entre copa e porta-enxerto. Enquanto a tangerina Sunki Tropical e o Híbrido 059 induziram as menores copas ao tangelo Page, assim como o citrumelo Swingle induziu menor volume de copa ao pomelo Flame, obtendo-se plantas mais compactas que podem facilitar o manejo quando adensadas, sendo alternativas para diversificar o cultivo de citros em condições similares.

\section{AGRADECIMENTOS}

Ao Conselho Nacional de Desenvolvimento Científico e Tecnológico (CNPq) pela concessão de bolsas, à Embrapa Mandioca e Fruticultura, à Embrapa Semiárido e à Secretaria de Agricultura de Russas (SEAGRI).

\section{REFERÊNCIAS}

Alvares, C. A., Stape, J. L., Sentelhas, P. C., Moraes, G., Leonardo, J., \& Sparovek, G. (2013). Köppen's climate classification map for Brazil. Meteorologische Zeitschrift, 22(6), 711-728.

Azevedo, F. A., Pacheco, C. D. A., Schinor, E. H., Carvalho, S. A., \& Conceição, P. M. (2015). Yield of Folha Murcha sweet orange grafted on Rangpur lime under high density planting. Bragantia, 74(2), 184-188.

Barbieri, R. L., \& Stumpf, E. R. T. (2008). Origem e evolução de plantas cultivadas (909 pp.). Brasília: Embrapa Informação Tecnológica.

Bastos, D. C., Passos, O. S., Ataíde, E. M., Sá, J. F., Girardi, E. A., \& Azevedo, C. L. L. (2014). Cultivo de citros no semiárido brasileiro (30 pp., Documentos, 266). Petrolina: Embrapa Semiárido.

Bastos, D. C., Passos, O. S., Calgaro, M., Ferreira, R. C. F., Silva, A. C. M., \& Rocha, E. M. M. (2017a). Pomeleiro Flame: nas condições do Vale do São Francisco (2 pp.). Cruz das Almas: Embrapa Mandioca e Fruticultura.

Bastos, D. C., Sombra, K. E. S., Andrade, H. M., Santos Filho, L. G., \& Passos, O. S. (2017b). Biometric evaluation of orange cultivars using different rootstocks in the semiarid region of Ceará, Brazil. Citrus Research \& Technology, 38(1), 71-76.

Bastos, D. C., Sombra, K. E. S., Loureiro, F. L. C., Silva, A. C. C., \& Passos, O. S. (2017c). Initial development of Tahiti acid lime trees on different rootstocks in the semiarid region of Ceará, Brazil. Citrus Research \& Technology, 38(1), 77-82.

Carvalho, L. M., Carvalho, H. W. L., Soares Filho, W. S., Martins, C. R., \& Passos, O. S. (2016). Porta enxertos promissores, alternativos ao limoeiro 'Cravo', nos Tabuleiros Costeiros de Sergipe. Pesquisa Agropecuária Brasileira, 51(2), 132-141. 
Coordenadoria de Defesa Agropecuária do Estado de São Paulo - CDA. (2017). Dados da citricultura do Estado de São Paulo por variedade base: $1^{\circ}$ Semestre 2017 (23 pp.). Campinas: CDA.

Cunha Sobrinho, A. P., Magalhães, A. F. J., Souza, A. S., Passos, O. S., \& Soares Filho, W. S. (2013). Cultura do Citros (vol. 1, 399 pp.). Brasília: Embrapa Informação Tecnológica.

Empresa Brasileira de Pesquisa Agropecuária-EMBRAPA. (2013). Sistema brasileiro de classificação de solos (3. ed. rev. ampl., 353 pp.). Brasília: Embrapa.

Fallahi, E., \& Rodney, D. R. (1991). Tree size, fruit quality, and leaf mineral nutrient concentration of Fairchild mandarin on six rootstocks. Journal of the American Society for Horticultural Science, 116(1), 2-5.

Lopes, J. M. S., Déo, T. F. G., Andrade, B. J. M., Giroto, M., Felipe, A. L. S., Sobrenome Junior, C. E. I., Bueno, C. E. M. S., Silva, T. F., \& Lima, F. C. C. (2011). Importância econômica do citros no Brasil. Revista Científica Eletrônica de Agronomia., 10(20), 1-3.

Loureiro, F. L. C., Sombra, K. E. S., Silva, A. C. C., Passos, O. S., \& Bastos, D. C. (2016). Avaliação biométrica de Pomeleiro 'star ruby'sob diferentes porta-enxertos no semiárido do Ceará. Revista Técnico-Científica do CREA-PR, 1(1), 1-9.

Oliveira, R. P., Souza, E. D. S., Scivittaro, W., Castro, L. A. S., \& Rocha, P. S. G. (2012). 'Flame': pomelo de polpa bem vermelha e sem sementes (2 pp.). Pelotas: Embrapa Clima Temperado.

Passos, O. S. (2012). Citrandarins: os porta-enxertos Indio, Riverside e San Diego (3 pp.). Cruz da Almas: Embrapa Mandioca e Fruticultura.

Passos, O. S., \& Soares Filho, W. S. (2003). Page CNPMF: opção para a citricultura de mesa (2 pp.). Cruz das Almas: Embrapa Mandioca e Fruticultura.

Passos, O. S., Soares Filho, W. S., \& Cunha Sobrinho, A. P. (2011a). Citrandarin 'Índio': nova opção de portaenxerto para a citricultura brasileira (2 pp.). Cruz da Almas: Embrapa Mandioca e Fruticultura.

Passos, O. S., Soares Filho, W. S., \& Cunha Sobrinho, A. P. (2011b). Citrandarin 'Riverside': nova opção de porta-enxerto para a citricultura brasileira (2 pp.). Cruz da Almas: Embrapa Mandioca e Fruticultura.

Passos, O. S., Soares Filho, W. S., \& Cunha Sobrinho, A. P. (2011c). Citrandarin 'San Diego': nova opção de porta-enxerto para a citricultura brasileira (2 pp.). Cruz da Almas: Embrapa Mandioca e Fruticultura.

Passos, O. S., Soares Filho, W. S., \& Peixouto, L. S. (2005). Variedades copa de citros para mesa (20 pp., Documentos, 154). Cruz das Almas: Embrapa Mandioca e Fruticultura.

Passos, O. S., Soares Filho, W. S., Cunha Sobrinho, A. P., Souza, A. S., Santos, L. C., \& Peixouto, L. S. (2016). Banco ativo de germoplasma de citros da Embrapa Mandioca e Fruticultura (6 pp). Cruz das Almas: Embrapa Mandioca e Fruticultura.

Portella, C. R., Marinho, C. S., Amaral, B. D., Carvalho, W. S. G., Campos, G. S., Silva, M. P. S., \& Sousa, M. C. (2016). Desempenho de cultivares de citros enxertadas sobre o trifoliateiro 'Flying Dragon' e limoeiro 'Cravo', em fase de formação do pomar. Bragantia, 75(1), 70-75.

Primavesi, A. (2014). Pergunte ao solo e às raízes: uma análise do solo tropical e mais de 70 casos resolvidos pela agroecologia (1. ed., 288 pp.). São Paulo: Nobel.

Rodrigues, M. J. S., Oliveira, E. R. M., Girardi, E. A., Ledo, C. A. S., \& Soares Filho, W. S. (2016). Citrus nursery tree production using different scion and rootstock combinations in screen house. Revista Brasileira de Fruticultura, 38(1), 187-201.

Silva, C. E. F., Gama, B. M. V., Oliveira, L. M. T. M., Araujo, L. T., Araujo, M. L., Oliveira Junior, A. M., \& Abud, A. K. S. (2016). Use of 'lime' orange and their wastes in the development of new products. Revista Brasileira de Engenharia de Biossistemas, 10(1), 69-96.

Silva, F. A. Z., \& Azevedo, C. A. V. (2016). The Assistat Software Version 7.7 and its use in the analysis of experimental data. African Journal of Agricultural Research, 11(39), 3733-3740.

Simonetti, L. M. (2015). Avaliação de novos híbridos de porta-enxertos para a laranjeira 'Valência' (Dissertação de mestrado). Universidade Estadual Paulista "Júlio de Mesquita Filho, Botucatu.

Sombra, K. E. S., Loureiro, F. L. C., Silva, A. C. C., Sombra Júnior, C. A., Passos, O. S., \& Bastos, D. C. (2017a). Desenvolvimento inicial de Tangerineira-Tangor Piemonte sobre diferentes porta-enxertos no Semiárido. In A. T. S. Alfaro, \& D. G. Trojan (Orgs.), Descobertas das ciências agrárias e ambientais 3 (pp. 172-180). Ponta Grossa: Atena Editora. 
Sombra, K. E. S., Loureiro, F. L. C., Silva, A. C. C., Sombra Júnior, C. A., Passos, O. S., \& Bastos, D. C. (2017b). Desenvolvimento inicial de limoeiro siciliano sobre diferentes porta-enxertos em espaçamento adensado no Semiárido do Ceará. In A. T. S. Alfaro, \& D. G. Trojan (Orgs.), Descobertas das ciências agrárias e ambientais 3 (pp. 163-171). Ponta Grossa: Atena Editora.

Sombra, K. E. S., Silva, A. C. C., Loureiro, F. L. C., \& Uchôa, C. N. (2018). A citricultura como instrumento de preservação da agricultura familiar no Semiárido Cearense, Brasil. Revista de Extensão e Estudos Rurais, 7(1), 353-372.
Teófilo Sobrinho, J., Pompeu Júnior, J., \& Figueiredo, J. O. (2012). Adensamento de plantio da laranjeira 'Valência' sobre Trifoliata - Resultados de 18 anos de colheita. Citrus Research \& Technology, 33(1), 49-58.

Recebido: Julho 23, 2018

Aceito: Outubro 11, 2018

Como citar: Sombra, K. E. S., Loureiro, F. L. C., Silva, A. C. C., Silva, M. P., Passos, O. S. \& Bastos, D. C. (2018). Avaliação biométrica de Tangelo Page e Pomelo Flame sobre diferentes porta-enxertos no semiárido do Ceará, Brasil. Citrus Research \& Technology, 39, e1038. https://doi. org/10.4322/crt.16418. 
